\title{
Recent Advances in Coherent Anti-Stokes Raman Scattering of Carbon Dioxide for Combustion Diagnostic. (Article Review)
}

\author{
Henry Misoi, Josephat Tanui, Patrick Wanjiru, and Paul Kioni
}

\begin{abstract}
The rising climatic degradation due to the emission of greenhouse gases is leading to emergence of clean combustion technology, oxy-fuel combustion to minimize the emissions of carbon dioxide into the atmosphere in combustion. Nitrogen molecules are used as probe molecule in laser-based combustion diagnostic in nitrogen rich air combustion. However, with the introduction of oxy-fuel combustion, carbon dioxide becomes the dominant molecule and has to be considered as probe molecule in combustion diagnostic. A detailed knowledge about thermodynamic properties: temperature, pressure and species concentration are necessary in optimization of combustion and minimizing the emission into the atmosphere. The non-intrusive spectroscopic measurement techniques are the most accurate methods to determine the combustion properties. The purpose of this review is to provide a brief overview of the recent advances made in application of coherent anti-Stokes Raman scattering of carbon dioxide for development of models for thermometry. However, there is no sufficient empirical data of time-domain S-branch Raman linewidth dependence on temperature that has been determined for pure-rotational coherent anti-Stokes Raman scattering of carbon dioxide and its mixtures for development of models for thermometry.
\end{abstract}

Index Terms - Combustion, Laser, Oxygen, Carbon Dioxide, Green House Gases, Fuel.

\section{INTRODUCTION}

There is a rising concern about climatic degradation in the world due to the massive emissions of greenhouse gases (GHG) that include carbon monoxide (CO), methane $\left(\mathrm{CH}_{4}\right)$, chlorofluorocarbons (CFCs), nitrogen oxides $\left(\mathrm{NO}_{\mathrm{x}}\right)$, and carbon dioxide $\left(\mathrm{CO}_{2}\right)$ [1]. $\mathrm{CO}_{2}$ is the major contributor to GHGs and it is the main product of combustion of hydrocarbon fuel [2], [3]. Fossil fuels are the most reliable source of energy in the world due to their flexibility and availability as compared to other conventional and renewable energy sources: biomass, solar, and wind energy. Fossil fuels drives the economy of the world and their use as a source of energy is inevitable even though it is the major hazard to the environment [4].

Oxy-fuel combustion is currently replacing air/fuel combustion in the development of the new technologies to minimize the emission of GHGs into the atmosphere. The new technologies have enabled the recirculation of flue gases in coal-fired power generation plants: It enables carbon capture and storage method [5], [6]. Nitrogen $\left(\mathrm{N}_{2}\right)$ is used as a probing molecule during air-fuel combustion diagnostic since it is an inert gas and is a dominant molecule in the air.

However, in oxy-fuel combustion, other molecules that include oxygen $\left(\mathrm{O}_{2}\right)$ and $\mathrm{CO}_{2}$ have to be considered due to the absence of $\mathrm{N}_{2}$. The introduction of the recirculation of exhaust gas in oxy-fuel combustion to control the combustion process makes $\mathrm{CO}_{2}$ the dominant molecule in the entire combustion process hence suitable for use as a probing medium. High temperatures are achieved with the use of pure oxygen without pre-heating the air during combustion and thus making it preferable in various applications such as in the glass production industry [7]. The use of $\mathrm{CO}_{2}$ as a makeup gas to replace $\mathrm{N}_{2}$ during coal combustion lowers the gas temperatures and lowers the burnout of char because of the high heat capacity of $\mathrm{CO}_{2}$ as compared to $\mathrm{N}_{2}$ [8]. Also, $\mathrm{CO}_{2}$ lowers the conversion of coal-nitrogen to NOx and emission of $\mathrm{CO}$ into the atmosphere [8], [9]. The detailed knowledge about the species concentration, pressure, and temperature of the combustion is required to optimize combustion efficiency and minimize the emission of $\mathrm{CO}_{2}$ gas into the atmosphere. The measurement of the combustion species can be done using intrusive and non-intrusive measurement methods. However, a non-intrusive method is preferred since it does not interfere with the combustion process. The non-intrusive method entails laser-based techniques such as: Laser-Induced Fluorescence (LIF), Rayleigh scattering, and coherent Raman scattering (CRS) [10]. The application of laser-based techniques has enabling a precise measurement of important combustion parameters. Since laser techniques are nonintrusive, the upper-temperature limit in the application does not exist and can be applied to both equilibrium and nonequilibrium phenomena [11], [12].

\section{RAMAN SCATTERING}

The Raman scattering is an inelastic scattering of an incident photon with frequency shift as shown in Fig.1. 




Fig. 1. Energy level diagram of vibrational Raman scattering.

The frequency shift of photon from lower to higher frequency leads to an increase in energy and is termed as coherent anti-Stokes Raman scattering (CARS). The frequency shift of a photon from a higher to a lower frequency leads to a decrease in energy and is termed as coherent Stokes Raman scattering (CSRS) [13]. CARS is widely in use for the measurement of temperature and concentration of the combustion species unlike CSRS that is strongly affected by fluorescence [14]. Raman scattering involves Raman transition between different energy levels which is either rotational or rovibrational. Rotational coherent anti-Stokes Raman spectroscopy (RCARS) occurs when the transition is between different rotational energy levels in the same vibration state (for pure RCARS). Vibrational coherent antiStokes Raman spectroscopy (VCARS) occurs when the transition is between different vibrational energy levels. RCARS has widely spaced spectral lines giving higher accuracy of thermometry at moderate and low temperatures when compared to vibrational CARS. Also, the temperatures have a weak dependence on the non-resonance susceptibility [15]. The rotational CARS give a high resolution of individual lines in S-branch spectra during measurements because of the wide separation between the adjacent lines [16]. There is no pressure collisional narrowing with RCARS because the wide separation helps in avoiding the effect of overlap arising from line broadening due to high collision frequencies at elevated pressure. There is no pressure collision narrowing effect and the spectral lines remain separated for up to at least $5 \mathrm{MPa}$ in acetylene /air fuel [17]. RCARS has successfully been used in temperature determination of sooting flame in air-fed hydrocarbon fuel and is almost free from spectral interference from $\mathrm{C}_{2}$ radicals that are produced by laser leading to errors in temperature evaluation [11], [18].

The rotational CARS is found to be more suitable for thermometry accuracy at lower temperature due to the high excitation of vibrational state at elevated temperatures. The vibrational CARS is significantly applied in thermometry at temperatures of approximately above $1000 \mathrm{~K}$ when the excited vibrational states are significantly populated and VCARS has the strongest temperature-sensitivity [17], [19]. Most of the research that has been conducted on CARS has been mostly on the vibrational CARS. However, the determination of the concentration of species using VCARS is largely affected by non-resonant background resulting in poor spectral sensitivity on concentration [15]. Also, the determination of the concentration of multiple species is not possible by VCARS because the spectral lines of different species are too far from each other in vibrational resonance. Vibrational energy levels are widely separated in VCARS more than rotational energy level in RCARS. However, the RCARS technique can be improved to apply to higher temperatures thermometry through the study and application of other combustion molecules. Multiple species can be detected and thermometry can be achieved at elevated temperatures with rotational CARS. Also, the non-resonant background is suppressed in time-domain RCARS so as to improve the accuracy, sensitivity and applicability [20], [21]. CARS has been applied primarily to $\mathrm{N}_{2}$ because $\mathrm{N}_{2}$ is present during combustion in high concentration and it is inert hence it is available during pre-combustion in air/fuel mixture and after combustion in exhaust gases. However, $\mathrm{N}_{2}$ is absent in combustion systems that do not use air-fuel environment hence other combustion species should be considered.

Carbon dioxide is a vital molecule in combustion diagnostic since it is a major product of conventional combustion. $\mathrm{CO}_{2}$ is a triatomic linear molecule with a larger rotational Raman cross-section as compared to $\mathrm{N}_{2}$ and $\mathrm{O}_{2}$ [22]. More emphasis on combustion has been on air-fuel combustion and the effect of $\mathrm{CO}_{2}$ on spectra has not been considered. In RCARS, the research that has been done has been mostly on diatomic molecules on $\mathrm{N}_{2}$ and $\mathrm{O}_{2}$ gas systems. Research has also been done on other polyatomic molecules that include $\mathrm{CH}_{4}, \mathrm{C}_{2} \mathrm{H}_{4}, \mathrm{C}_{2} \mathrm{H}_{6}, \mathrm{H}_{2} \mathrm{O}, \mathrm{H}_{2}$ and $\mathrm{CO}$ [23-26]. Other molecules that are considered for combustion diagnostics include $\mathrm{C}_{2} \mathrm{H}_{2}$ and $\mathrm{N}_{2} \mathrm{O}$ (linear polyatomic molecules) [17], [27]. More quantitative investigation has to be done on RCARS regarding $\mathrm{CO}_{2}$ molecules for the determination of combustion parameters. The research has been restricted to $\mathrm{N}_{2}, \mathrm{O}_{2}, \mathrm{H}_{2}, \mathrm{C}_{2} \mathrm{H}_{4}$, and $\mathrm{C}_{2} \mathrm{H}_{6}$ gas system in combustion environment using nitrogen-rich air from the atmosphere since at moderate concentration of $\mathrm{CO}_{2}$ and very high temperature the spectra intensity of $\mathrm{CO}_{2}$ is comparatively small compared to $\mathrm{N}_{2}$ spectra [28].

\section{THE CARS PROCESS FOR THERMOMETRY ON $\mathrm{CO}_{2}$}

The Rayleigh and coherent Raman scattering occur as a result of the existence of oscillating induced polarization. Rayleigh scattering involves elastic scattering where there is no frequency shift. In Raman scattering, there is a frequency shift from the incident beam with regard to rotational or vibrational frequency [11]. New parameter, CARS and CSRS signals occur simultaneously and are stronger than conventional Raman scattering by many orders of magnitude and can be completely collected because of its coherence. The generated beam through inelastic scattering either has a higher or lower frequency than the probe beam. The beam with a higher frequency is shifted in anti-Stokes direction while the beam with lower frequency is shifted in Stokes direction.

There are at least two beams in CARS (light scattering) that are focused to a common point of intersection (scattering region) in the sample where a particular behaviour of 
ensemble of molecules is picked by a certain characteristic signal within the volume of interaction [30]. Also, CARS form a nonlinear four-wave mixing system with laser beams having different frequencies. The three laser photons involved are: pump, Stokes, and probe photons that interact with the sample leading to emergence of a nascent coherent optical signal. The difference between the pump and the Stokes beam gives a rotational or vibrational coherent Raman excitation in the probed molecules. There is an induction of oscillating polarization with frequency as expressed in Eqn. (1).

$$
\omega_{R}=\omega_{\text {pump }}-\omega_{\text {Stokes }}
$$

where $\omega_{R}$ is the Raman frequency shift and $\omega_{\text {pump }}$ and $\omega_{\text {Stokes }}$ are the frequencies of pump and Stokes laser beams, respectively. The CARS signal at frequency $\omega_{\text {CARS }}$ originates from the sum of the Raman frequency and the probe laser beam $\omega_{\text {probe }}$. The frequency $\omega_{\mathrm{CARS}}$ is given by the equation of the law of conservation of energy and momentum in the form of frequency since the energy of a wave is proportional to its frequency as in Eqn. (2). The molecules can either undergo rotational transition or vibrational transition when excited by photons. Rotational and vibrational transition involves the excitation of molecules from lower to higher rotational and vibrational energy levels respectively. The rotational and vibrational transition term value of $\mathrm{CO} 2$ is $1.56 \mathrm{~cm}^{-1}$ and $667 \mathrm{~cm}^{-1}$, respectively. The vibrational transition energy is higher than rotational transition energy since the energy gap between two consecutive energy levels is bigger. Also, several rotational energy levels are available within a particular vibrational energy level [14], [31]-[33]. The anti-Stokes signal does not get fluorescence interferences for the most part making it suitable for thermometry. CARS technique has high sensitivity and is capable of detecting flame radicals at trace concentrations even in a hostile combustion environment [10]. Coherent Stokes Raman spectroscopy (CSRS) involves interaction between two or more signals in the sample leading to the generation of Stokes frequency beam that is lower in energy than the probe beam as shown by Fig.1. CSRS is affected by fluorescence interference and it is not often used in combustion diagnostics.

\section{FOUR-WAVE MIXING}

Four-wave mixing entails a non-linear optic phenomenon where three electromagnetic waves interact to produce a fourth wave as shown in Fig. 2.

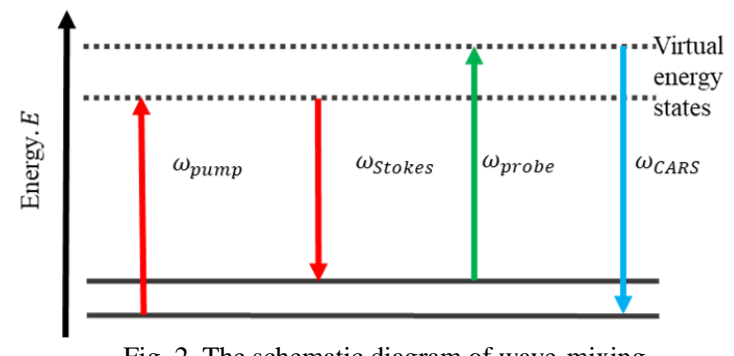

Fig. 2. The schematic diagram of wave-mixing.
The break in the axis indicates the high level of energy in the probe and CARS beam. The wave is generated as a result of photon scattering due to third-order non-linearity of the medium of interaction [34]. The continuous line in the figure indicates the transitions for Stokes Raman scattering. The upward red and green arrows indicate the exciting pump and probe beam with frequencies $\omega_{\text {pump }}$ and $\omega_{\text {probe }}$ respectively. The downward red arrow indicates the Stokes transition with frequency $\omega_{\text {Stokes }}$. The gas molecules undergo transition from lower to higher energy state. The CARS occur when the Stokes beam is populating the excited state and the probe beam, through the emission of anti-Stokes radiation, brings back the molecules to the lower energy state [30]. The beam generated from the scattering of the probe signal remains coherent when the law of conservation of energy and momentum is maintained as shown in Eqn. (2).

$$
\omega_{\text {CARS }}=\omega_{\text {pump }}-\omega_{\text {Stokes }}+\omega_{\text {probe }}
$$

The intense electric fields of the light beam induce polarization of molecules and thus cause oscillation of dipoles [35]. The induced dipoles are not the eigenstate of the molecule but virtual states. Thus, the system is excited and de-excited immediately during pump and Stokes wave mixing.

\section{POLARIZATION AND CARS SignAL STRENGTH}

The electron distribution of molecules and atoms are distorted to some extent when subjected to an external electric field resulting in the induction of dipole moments [36]. The susceptibility of the medium, which is a third order non-linear optical phenomenon depends upon the induced polarization in the medium. The polarizability of the molecules determines the extent to which the electric field distorts the electron distribution in the molecules. The susceptibility is the measure of how easy the molecules can be polarized and is dependent on the position of the nucleus in the molecules [11]. The linear susceptibility of the medium characterizes medium index of refraction since it is related to the dielectric constant.

The higher-order susceptibility terms are weaker when compared to linear terms and are considered when there is an electric field with considerable strength because of the presence of dominant resonant shift in the nonlinear susceptibilities. The even terms vanish because of the inversion symmetry properties in the gas medium (isotropic media) and thus the third-order forms the lowest order of nonlinearities [11], [30]. The fifth and subsequent odd orders of nonlinearities are very weak and are not considered.

\section{A. Dual Broadband Approach}

A broadband beam has energy at a wide range of frequencies that allows for the population of the multiple rotational Raman coherences. At this stage, a single probe can be used to probe every excited rotational state and generate the CARS signal as shown in Fig. 3. 


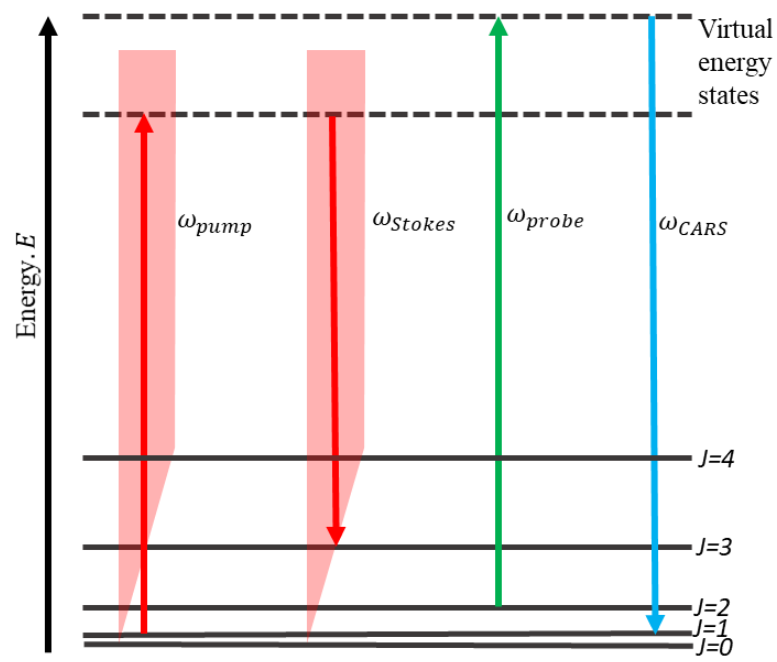

Fig. 3. Dual-broadband energy diagram of rotational CARS with the selection rule $\Delta \mathrm{J}=2$, (S-branch).

The red arrows represent the pump and Stokes pulse, the green arrow represents probe pulse, and the blue arrow represents the CARS pulse. The wedged arrows represent broadband pulses. Dotted lines represent a virtual energy state. Different molecules have different energy levels and the difference between the pump and the Stokes beam frequencies yields rotational or combination of rotational and vibrational excitations. The frequency difference of the pulses can be altered to bring about a complete Raman spectrum. The spectral analysis of a single molecular species can be carried out when other species have been suppressed by selecting a particular wavelength in rovibrational CARS. Dual-broadband RCARS can also be used in the analysis of multispecies where broadband pump and Stokes beams are used. The temperature, pressure, and concentration parameters of combustion are accessible based on the singleshot by the use of broadband CARS with nanosecond time resolution [33], [37].

\section{LINE BROADENING}

The width of Raman lines is attributed to the Doppler effect, pressure effect, limited instrumental resolution, and superposition of the ground state spectrum by hot band transitions [30]. The transition of the molecules has a finite frequency linewidth that makes it essential during diagnostic. The linewidth, relative intensity as well as the temporal behavior has information about the molecular ensemble. The position of the spectral lines, their relative positions, and their intensities are applied for thermometry, species concentration, and measurement of pressure.

The excited quantum state has a natural lifetime before undergoing spontaneous decay. According to the Heisenberg uncertainty principle, the natural linewidth depends on the energy [38]. The natural linewidth cannot be determined precisely since it is relatively smaller than line broadening that results from either pressure broadening or Doppler broadening [39]. The energy profile broadens with the shortening of the lifetime of the excited state resulting in a line shape described by Lorentzian expression [34].

Collision broadening is a homogeneous broadening that results from the collision between molecules or atoms.
Inelastic collision, only kinetic energy changes and there is no effect in the internal energy of the molecules leading to change in the velocity. In inelastic conditions, there are changes in internal energy where the change in kinetic energy is transferred to or from rotational or vibrational energies of the molecules. The rate of collision increases with the increase in the medium pressure and thus also called pressure broadening. The energy transition favours resonant transition hence it depends on rotational and vibrational energy levels of the molecules. The phase of the emitted radiation changes when collision occurs during its emission. The emitted beam has finite wave train with mean duration $\tau_{\mathrm{c}}$ and the random phase shift due to collisions. The Lorentzian line shape profile shown in Eqn. (1) is obtained through its Fourier analysis:

$$
I(\omega)=I_{0} \frac{\left(\gamma / 2+N . \bar{v} \sigma_{b}\right)^{2}}{\left(\omega-\omega_{0}-N_{B} v \sigma_{S}\right)^{2}+\left(\gamma / 2+N \cdot v \sigma_{b}\right)^{2}}
$$

where $\mathrm{N}$ is the total density of molecules, and $\mathrm{v}$ is the average relative velocity and NB is the number of density of molecules B. The FWHM $\Delta \omega \mathrm{c}$ is derived from mean collision time. The linewidth can also be derived from the MaxwellBoltzmann's velocity distribution law of statistical thermodynamics as shown in Eqn. (4).

$$
\Delta \omega_{c}=\sqrt{\frac{3}{4 m k T} \cdot d^{2} p}
$$

where $\mathrm{m}$ is the mass of the molecule, $\mathrm{T}$ is the temperature of the medium, $\mathrm{d}$ is the diameter of the molecule, and $\mathrm{p}$ is the pressure of the medium. Collision broadening is quite larger than natural broadening.

The collisional broadening is a predominant factor for the experiments conducted at atmospheric pressure [30]. The collisions in the ensembled molecules are described by Eqn. (5) based on the semi-classical description of the density function theorem (DFT) formulation.

$$
i \hbar \frac{d \widehat{\rho}}{d t}=[\widehat{H} \cdot \hat{\rho}]-\hat{\Gamma} \cdot \hat{\rho}
$$

where $\Gamma$ is the damping term, $\rho^{\wedge}$ is the electron density and $\mathrm{H}^{\wedge}$ is the Hamiltonian constant. The framework of the impact theory is used to solve the Liouville equation, Eqn. (5) for a transition $J^{\prime} \rightarrow J$ " based on the assumption that the collision time is shorter than the time between collisions. The thirdorder non-linear susceptibility takes the form of expression given in Eqn. (6).

$$
\chi_{C A R S}^{(3)}=-\frac{1}{\hbar} \vec{\alpha} \underline{\underline{G}}(\omega)^{-1} \Delta N \vec{\alpha}+\chi_{N R}
$$

where $\sim \alpha$ is the vector transition amplitude, $G(\omega)$ is the Gordon matrix, $\Delta \mathrm{N}$ is the population difference between energy levels and $\chi \mathrm{NR}$ is the nonresonant susceptibility component. The Gordon matrix contains encoded Ramans linewidth (HWHM) in its diagonal elements $\left(\mathrm{J}^{\prime} \rightarrow \mathrm{J}^{\prime}\right)$ [40]. The modified energy gap (MEG) law rely on the Galantry rather than Lorentzian or Voigt line shape as suggested by Rahn and Palmer [41]. MEG law correctly includes Dicke narrowing of the spectral lines resulting from a change in 
velocity as a result of collisions [42]. Dicke narrowing affects the contribution of Doppler effect at elevated pressures and the transition rate is shown by Eqn. (7) [43].

$$
-k_{J^{\prime} J^{\prime \prime}}=\alpha p \frac{1-e^{-m}}{1-e^{\frac{m T}{T_{0}}}}\left(\frac{T_{0}}{T}\right)^{n}\left(\frac{1+\frac{\alpha E_{i}}{k T \delta}}{1+\frac{\alpha E_{i}}{k T}}\right)^{2} \exp \left(-\frac{\beta \Delta E_{i j}}{k T}\right)
$$

where $\alpha, \beta, \delta$ and $\mathrm{n}$ are fixed fitting parameters, $\mathrm{p}$ is the pressure and $\mathrm{T}_{0}$ is the reference temperature [30]. The MEG law is used to compute a spectral library for different temperatures with known parameters. The experimental spectra are fitted with the theoretical spectra from the library where the temperature is obtained from the best-fitting theoretical spectrum.

\section{THE CARS PROCESS FOR THERMOMETRY ON $\mathrm{CO}_{2}$}

From the research that has been done, the comparison of $\mathrm{CO}_{2}$ and $\mathrm{N}_{2}$ spectra at $300 \mathrm{~K}, 0.1 \mathrm{MPa}$ and $15 \% \mathrm{CO}_{2}$, it was found that $\mathrm{CO}_{2}$ displayed narrower line spacing than $\mathrm{N}_{2}$ because of its higher molecular weight [30], [37]. The transition linewidth of $\mathrm{CO}_{2}$ was found to be larger than the corresponding linewidth of $\mathrm{N}_{2}$ [37]. Through the determination of $\mathrm{CO}_{2}$ concentration in the mixture (probe volume), the sensitivity of the pure rotational CARS can be increased significantly especially at high pressure [15], [32]. The $\mathrm{CO}_{2}$ spectral lines melt together forming a common envelope with the rising particle density at pressure beyond $10 \mathrm{MPa}$ and room temperature. With the constant volume of $\mathrm{CO}_{2}$, it was found that the contribution of relative intensity by $\mathrm{CO}_{2}$ to spectral appearance increases significantly with the increase in pressure with respect to $\mathrm{N}_{2}$ and $\mathrm{O}_{2}$ [32], [43].

$\mathrm{CO}_{2}$ molecules obey the Bose-Einstein statistics and thus the number of fermions in one side of the axis is zero. The states of odd $\mathbf{J}$ do not exist and only the states of even $\mathbf{J}$ exist at the ground electronic state. The selection rule thus allows for the transition that involves only $\Delta \mathrm{J}=+2$ that occurs as a result of the symmetry argument of the polarizability [30], [44].

The research on validation of RCARS spectroscopy model for $\mathrm{CO}_{2}$ thermometry has been performed in premixed $\mathrm{CO}_{2}$ /air flame (equivalent ratio of 0.8 to 2.5) with $\mathrm{N}_{2}$ and $\mathrm{CO}_{2}$ RCARS model at atmospheric pressure [19], [32]. It was found that the spectral lines increase in number with the increase in temperature and are shifted to higher Raman shift. The accuracy of temperature measurement with CARS was found to be good for the interval 294-1143 K with the error of being within $10 \mathrm{~K}(2 \%)$. The standard deviation was found to be increasing almost linearly with temperature for single mode Nd:YAG laser. The $\mathrm{CO}_{2}$ spectra envelopes below 150 $\mathrm{cm}^{-1}$ during evaluation of the high temperatures of the flame. The changes are attributed to uncertainties in the rotational linewidth and the assumptions that was made for the model. The spectral CARS increase the sensitivity at flame temperatures [19], [32].

The development of knowledge of $\mathrm{CO}_{2}$ spectroscopy and theoretical models for RCARS is essential for the development of the technique with potential of thermometry and detection of species over extensive conditions of oxy-fuel combustion. Further research is needed to extend the limit of thermometry.

\section{CONCLUSION}

The time-domain, pure-rotational Raman linewidths of self-broadened carbon dioxide at room temperature has been studied with pure-RCARS. However, the temperature dependent pure-rotational Raman linewidths data is not available for thermometry. The S-branch Raman linewidths of pure $\mathrm{CO}_{2}$ and its mixtures has been evaluated by applying the Modified Energy Gap (MEG) law but using the Q-branch linewidths which could cause an error in the evaluation. Therefore, research on S-branch Raman linewidths of $\mathrm{CO}_{2}$ at combustion temperature has to be conducted to avail the empirical data for calculation of $\mathrm{CO}_{2}$-RCARS spectra and its application to thermometry. Therefore, a precise calculation based on accurate S-branch linewidth will help in future to minimize the temperature measurement error.

\section{REFERENCES}

[1] S. T. IPCC, "Climate change 2014: Synthesis report," Contribution of Working Groups I, II and III to the Fifth Assessment Report of the Intergovernmental Panel on Climate Change, IPCC, Geneva, Switzerland, $151 \mathrm{pp}, 2014$.

[2] M. G. Hardik, "Effect of Global Warming," International Journal of Research in all Subjects in Multi Languages, vol. 2, no. 8, 2014.

[3] C. Wang, X. Zhang, Y. Liu, and D. Che, "Pyrolysis and combustion characteristics of coals in oxyfuel combustion," Applied Energy, vol. 97, pp. 264-273, 2012, doi: 10.1016/j.apenergy.2012.02.011.

[4] J.C.M. Pires, F. G. Martins, M.C.M. Alvim-Ferraz, and M. Simões, "Recent developments on carbon capture and storage: An overview," Chemical Engineering Research and Design, vol. 89, no. 9, 2011, doi: 10.1016/j.cherd.2011.01.028.

[5] B.J.P. Buhre, L. K. Elliott, C. D. Sheng, R. P. Gupta, and T. F. Wall, "Oxy-fuel combustion technology for coal-fired power generation," Progress in Energy and Combustion Science, vol. 31, no. 4, pp. 283 307, 2005, doi: 10.1016/j.pecs.2005.07.001.

[6] C. R. Shaddix and A. Molina, "Particle imaging of ignition and devolatilization of pulverized coal during oxy-fuel combustion," Proceedings of the Combustion Institute, vol. 32, no. 2, pp. 20912098, 2009, doi: 10.1016/j.proci.2008.06.157.

[7] J. W. Tröger, C. Meißner, and T. Seeger, "High temperature $\mathrm{O}_{2}$ vibrational CARS thermometry applied to a turbulent oxy-fuel combustion process," J. Raman Spectrosc., vol. 47, no. 9, pp. 11491156, 2016, doi: 10.1002/jrs.4937.

[8] H. Lie, R. Zailan, and B. Gibbs, "Comparisons of pulverized coal combustion in air and in mixtures of $\mathrm{O}_{2} / \mathrm{CO}_{2}$, , Fuel, vol. 84, 7-8, pp. 833-840, 2005, doi: 10.1016/j.fuel.2004.11.018.

[9] Z.-J. Wu, X. Yu, L.-Z. Fu, J. Deng, Z.-J. Hu, and L.-G. Li, “A high efficiency oxyfuel internal combustion engine cycle with water direct injection for waste heat recovery," Energy, vol. 70, pp. 110-120, 2014, doi: 10.1016/j.energy.2014.03.095.

[10] M. J. Evans and P. R. Medwell, "Understanding and Interpreting Laser Diagnostics in Flames: A Review of Experimental Measurement Techniques," Front. Mech. Eng., vol. 5, 2019, doi: 10.3389/fmech.2019.00065.

[11] A. C. Eckbreth, Laser Diagnostics for Combustion Temperature and Species: Gordon and Breach Publishers, 1996.

[12] C. Brackmann, J. Bood, M. Afzelius, and P.-E. Bengtsson, "Thermometry in internal combustion engines via dual-broadband rotational coherent anti-Stokes Raman spectroscopy," 2004, doi: 10.1088/0957-0233/15/3/R01).

[13] L. J. Jacob and H.-P. Deigner, "Nanoparticles and Nanosized Structures in Diagnostics and Therapy," in Precision Medicine: Elsevier, 2018, pp. 229-252.

[14] Y. Gao, A. Bohlin, T. Seeger, P.-E. Bengtsson, and C. J. Kliewer, "In situ determination of $\mathrm{N}_{2}$ broadening coefficients in flames for rotational CARS thermometry," Proceedings of the Combustion Institute, vol. 34, no. 2, pp. 3637-3644, 2013, doi: 10.1016/j.proci.2012.05.010. 
[15] L. Martinsson, P.-E. Bengtsson, M. Aldén, S. Kröll, and J. Bonamy, "A test of different rotational Raman linewidth models: Accuracy of rotational coherent anti-Stokes Raman scattering thermometry in nitrogen from 295 to $1850 \mathrm{~K}$," The Journal of chemical physics, vol 99, no. 4, pp. 2466-2477, 1993, doi: 10.1063/1.466197.

[16] E. Nordström, A. Hosseinnia, C. Brackmann, J. Bood, and P.-E. Bengtsson, "Raman linewidth measurements using time-resolved hybrid picosecond/nanosecond rotational CARS," Optics letters, vol 40, no. 24, pp. 5718-5721, 2015, doi: 10.1364/OL.40.005718.

[17] J. Bood, P.-E. Bengtsson, and M. Alden, "Temperature and concentration measurements in acetylene-nitrogen mixtures in the range 300-600 K using dual-broadband rotational CARS," Appl. Phys. B, vol. 70, pp. 607-620, 2000, doi: 10.1007/s003409900168.

[18] P.-E. Bengtsson, L. Martinsson, M. Aldén, and S. Kröll, "Rotational CARS Thermometry in Sooting Flames," Combustion Science and Technology, vol. 81, 1-3, pp. 129-140, 1992, doi: 10.1080/00102209208951797.

[19] F. Vestin, D. Sedarky, R. Collins, M. Alden, M. Linne, and P. Bengtsson, "Rotational coherent anti-Stokes Raman spectroscopy (CARS) applied to thermometry in high-pressure hydrocarbon flames," Combustion and Flame, vol. 154, 1-2, pp. 143-152, 2008, doi: 10.1016/j.combustflame.2007.10.014.

[20] S. Roy and T. R. Meyer, "Broadband coherent anti-Stokes Raman scattering spectroscopy of nitrogen using a picosecond modeless dye laser," Optics letters, vol. 30, no. 23, 2005, doi: 10.1364/OL.30.003222.

[21] Y. Ouazzany, J.P. Boquillon, and H.W. Schrötter, "High resolution CARS spectrum and analysis of the v1 band Q-branch of carbon dioxide," Molecular Physics, vol. 63, no. 5, pp. 769-777, 1988, doi: 10.1080/00268978800100551.

[22] S. Roy et al., "Direct measurements of collisionally broadened Raman linewidths of $\mathrm{CO}_{2}$ S-branch transitions," The Journal of chemical physics, vol. 138, no. 2, p. 24201, 2013, doi: 10.1063/1.4774093.

[23] W. D. Kulatilaka, J. R. Gord, and S. Roy, "Effects of $\mathrm{O}_{2}-\mathrm{CO}_{2}$ polarization beating on femtosecond coherent anti-Stokes Raman scattering (fs-CARS) spectroscopy of $\mathrm{O}_{2}$," Appl. Phys. B, vol. 102, no. 1, pp. 141-147, 2011, doi: 10.1007/s00340-010-4188-2.

[24] M. Afzelius, C. Brackmann, F. Vestin, and P.-E. Bengtsson, "Pure rotational coherent anti-Stokes Raman spectroscopy in mixtures of $\mathrm{CO}$ and $\mathrm{N}_{2}, "$ Applied optics, vol. 43, no. 36, 2004, doi 10.1364/AO.43.006664

[25] M. Afzelius et al., "Dual-broadband rotational CARS modelling of nitrogen at pressures up to $9 \mathrm{MPa}$. II. Rotational Raman line widths," Applied Physics B: Lasers and Optics, vol. 75, 6-7, pp. 771-778, 2002, doi: 10.1007/s00340-002-1020-7.

[26] S. Roy, J. R. Gord, and A. K. Patnaik, "Recent advances in coherent anti-Stokes Raman scattering spectroscopy: Fundamental developments and applications in reacting flows," Progress in Energy and Combustion Science, vol. 36, no. 2, pp. 280-306, 2010, doi: 10.1016/j.pecs.2009.11.001

[27] P. S. Hsu, H. U. Stauffer, N. Jiang, J. R. Gord, and S. Roy, "Direct measurements of collisional Raman line broadening in the S-branch transitions of acetylene (C2H2)," The Journal of chemical physics, vol. 139, no. 15, p. 154201, 2013, doi: 10.1063/1.4824859.

[28] A. Hosseinnia, C. Brackmann, and P.-E. Bengtsson, "Pure rotationa coherent anti-Stokes Raman spectroscopy of ethylene, experiments and modelling," vol. 234, pp. 24-31, 2019, doi: 10.1016/j.jqsrt.2019.05.022.

[29] M. Quack, High-resolution Raman Spectroscopy of Gases Chichester: Wiley, 2011. [Online]. Available: http:// onlinelibrary.wiley.com/book/10.1002/9780470749593

[30] R. K. Hanson, R. M. Spearrin, and C. S. Goldenstein, Spectroscopy and Optical Diagnostics for Gases. Cham: Springer International Publishing, 2016.

[31] H. F.R.S. Gerhard, Molecular Spectra and Molecular Structure: Spectra of Diatomic Molecules. New York: D.van Nostrand Company, INC, 1950.

[32] M. Schenk, S. Thomas, and A. Leipertz, "Time-resolved $\mathrm{CO}^{2}$ thermometry for pressures as great as $5 \mathrm{MPa}$ by use of pure rotational coherent anti-Stokes Raman scattering.," Applied optics, vol. 44, no. 31, 2005, doi: 10.1364/AO.44.006526.

[33] K. F. Renk, Basics of Laser Physics. Cham: Springer International Publishing, 2017.

[34] T. Seeger, J. Jonuscheit, M. Schenk, and A. Leipertz, "Simultaneous temperature and relative oxygen and methane concentration measurements in a partially premixed sooting flame using a novel CARS-technique," Journal of Molecular Structure, 661-662, pp. 515-524, 2003, doi: 10.1016/j.molstruc.2003.09.009.
[35] F. Bretislav and H. Dudley, "Polarization of Molecules Induced by Intense Nonresonant Laser Fields," The Journal of chemical physics, vol. 99, no. 42, 1995, doi: 10.1021/j100042a051.

[36] F. Vestin and P.-E. Bengtsson, "Rotational CARS for simultaneous measurements of temperature and concentrations of $\mathrm{N}_{2}, \mathrm{O}_{2}, \mathrm{CO}$, and $\mathrm{CO}_{2}$ demonstrated in a CO/air diffusion flame," Proceedings of the Combustion Institute, vol. 32, no. 1, pp. 847-854, 2009, doi 10.1016/j.proci.2008.06.027.

[37] M. J. Hollas, Modern Spectroscopy: John Wiley \& Sons, Ltd, 2004.

[38] H. J. Eichler, J. Eichler, and O. Lux, Lasers: Basics, Advances and Applications. Cham: Springer International Publishing, 2018.

[39] F. M. Porter, D. A. Greenhalgh, P. J. Stopford, D. R. Williams, and C. A. Baker, "A study of CARS nitrogen thermometry at high pressure," Appl. Phys. B, vol. 51, no. 1, pp. 31-38, 1990, doi: 10.1007/BF00332321.

[40] L.A. Rahn and R.E. Palmer, "Studies of nitrogen self-broadening at high temperature with inverse Raman spectroscopy," vol. 3, no. 9, 1986.

[41] G. Millot, B. Lavorel, and G. Fanjoux, "Pressure Broadening, Shift, and Interference Effect for a Multiplet Line in the Rovibrational Anisotropic Stimulated Raman Spectrum of Molecular Oxygen," Journal of Molecular Spectroscopy, vol. 176, no. 1, pp. 211-218, 1996, doi: 10.1006/jmsp.1996.0078.

[42] T. Seeger, F. Beyrau, A. Bräuer, and A. Leipertz, "High-pressure pure rotational CARS: comparison of temperature measurements with $\mathrm{O}_{2}$ $\mathrm{N}_{2}$ and synthetic air," J. Raman Spectrosc., vol. 34, no. 12, pp. 932 939, 2003, doi: 10.1002/jrs.1094.

[43] M. Schenk, T. Seeger, and A. Leipertz, "Simultaneous and timeresolved temperature and relative $\mathrm{CO}_{2}-\mathrm{N}_{2}$ and $\mathrm{O}_{2}-\mathrm{CO}_{2}-\mathrm{N}_{2}$ concentration measurements with pure rotational coherent anti-Stokes Raman scattering for pressures as great as $5 \mathrm{MPa}$," vol. 44, no. 26 , 2005, doi: 10.1364/AO.44.005582.

[44] N.C. Banwell and E.M. McCash, Fundamentals of molecular spectroscopy: (Vol. 851). New York: McGraw-Hill. New York: McGraw-Hill, 1994. 